\title{
Nutrición parenteral en Pediatría I. Fisiología y técnica
}

Drs. Y. H. Pacheco, A, Valdivieso, ${ }^{* *}$ F. Ruza** y M. C. Martinez.**

\begin{abstract}
After a historical review we analyse the metabulic bagis of caloric reguirements, and the reyuirements of carbor hidrates, tats and proleins, trace rlements and vitantins.

We analyse certain metabolit peculianities in parenteral nutrition: rensl solute overhoad. and os morality of solutions. Technical aspects in parenteral nutrition (central and peripherali) are explained. The cuntrol and management of parenteral autrition are exposed. Finally. the technical and metabolic complications of parenteral mutrition are detailed. Key words: Parenteral Nutrition. Metabolic Basis in. Techniques of, Complications in.
\end{abstract}

Entendemos por nutrición parenteral la administración por vía endovenosa de nutrientes en cantidad suficiente y equilibrada, para "aportar las necesidades calorificas diarias, corregir los déficits previos y cubrir las necesidades extraordinarias que en su caso se pueden producir". Su empleo está justificado solamente cuando sea "imposible, inadecuada o peligrosa la alimentación por vía digestiva", bien como alimentación oral o bien como alimentacion enteral continua, mediante sonda nasogástrica, gastrostomía o sonda duodenal.

La nutrición parenteral se puede realizar téenicamente de diversas formas. En función de ello existen diferentes clasificaciones, para poder etiquetarla más exactamente: ${ }^{\text {LI }}$

\subsection{Según el tipo de nutrición parenteral} realizada:

Alimentación purenteral exclusiva, cuando todos los nutrientes aportados al organismo se administran solamente por vía venosa, sin ningún tipo de alimentación oral. Esta es la auténtica alimentación parenteral.

\section{Alimentación parenteral complementaria o}

\footnotetext{
* Servicio de Pediatría, Huspital Guillermu Grant B.. de Concepción.

Departanaente de Pediatria, Universidad de Concepción (Chile!.

*"Clínica lnfantil "La Paz". Seccinon de Cuidadus Intensivor Pediátricos. Universidad Autimma Facultad de Medicina. Mudrid, España.
}

mixta, cuando los aportes IV colaboran con los suministrados por vía digestiva para alcanzar el total de calorias de la alimentación. Esta modalidad se realiza siempre en la fase de retirada de una A.P. exclusiva, tiene una amplia utilidad en ocasiones de necesidades extraordinarias y/o cuando la alimentación digestiva resulta peligrosa o dificultosa.

\subsection{Según la técnica (vena utilizada):}

Alimentación parenteral central: Cuando la punta del catéter que utilizamos para la alimentación la situamos en aurícula derecha o en vena cava superior.

Alimentación parenteral periférica: Cuando utilizamos cualquier vena periférica, en la que perfundimos los nutrientes mediante simple punción venusa.

1.3. Según el tipo de nutrientes suministrados:

Alimentación parenteral completa: Cuando administramos todos los nutrientes que normalmente constituyen una a dimentación equilibrada (príncipios inmediatos, minerales, oligoelementos y vitaminas).

Alimentación parenteral parcial o incompleta: Cuando se suministra un nutriente determinado (pérdida específica) o una dieta endovenosa incompleta para cubrir un corto espacio de tiempo o una función orgánica específica alterada (fallo renal agudo, etc.). 


\section{Antecedentes históricos}

El intento de administrar alimentación por vía venosa data del siglo XVII. Sir Christopher W'ren fue el primero en introducir sustancias nutritivas en venas de un perro, tras el descubrimiento de la circulación sanguínea por William Harvey en $1616 .{ }^{(2)}$ Posteriormente, Claude Bernard, en 1843, introduce, por primera vez, soluciones de azúcar en animales.'2! En 1873, Hodder inyecta leche por vía venosa en 3 enfermos de cólera, de los que se recuperó uno.1.3 En 1896, Biedl y Kraus emplean solución de dextrosa en humanos. ${ }^{1 /}$ No es muy lejana la lecha desde la que se emplea con seguridad la administraciún intravenosa de glucosa, tras el descubrimientu de los conceptos básicos de microbiologia por Pasteur y la subsecuente aplicación de los principios de asepsia y antisepsia por Lister.'t?

Èn relación coun el uso de proteína, Henriques y Anderson, en 1913, fueron los primeros en usar aminoácidos con éxitu en la alimentación parenteral, produciendo un balance nitrogenado positivo en animales mediante infusión de músculo digerido por extractu pancreático y agregado de glucosa, NaCl y KCJ."3r Posteriormente, Elmans, en 1939, y Riegel y col., en 1940, emplean una solución de hidrolizado de caseína junto a dexirosa en el hombre. ${ }^{(t,+,}$, t) El empleo de estos hidrolizados de caseína y de fibrina es rápidamente aceptado. Posteriormente es reemplazado por la fabricación de aminoácidos sintéticos.

La administración de emulsiones lipídicas fue empleada por primera vez por Halt y col. en 1935. ${ }^{\text {51 }}$ A finales de $1940 \mathrm{y}$ comienzos de 1950 , Siren nuevamente las usa. ${ }^{1 /}$ En 1964 son retiradas del mercado debido a las reacciones adversas producidas por la administración prolongada de un producto preparadu de infusión de aceite de semilla de algodón." Actualmente se han obtenido (por la escuela europea) productos a base de emulsiones de aceite de soja: Intralipid, que tiene muy buena tolerancia cuando es usado con las debidas precauciones. ${ }^{\text {6. 7. 3) }}$

En 1967, Dudrick describe la téenica que permite la administración venosa central de soluciones de glucosa y proteínas con una elevada osmolaridad, junto a los requerimientos de electrólitos y vitaminas. ${ }^{19,10,11}, 12,13,14,15$,

Ultimamente se está avanzando en la correcta administración de oligoelementos, como $\mathrm{Cu}, \mathrm{Zn}$, Co, Mn, etc., a los que cada vez se confiere mayor importancia metabólica. ${ }^{16,17}$ ?

Hoy día se emplea también la vía periférica con soluciones de menor osmolaridad. "18! Esta técnica podria incluso reemplazar la vía central, al dar una alimentación parenteral completa - con aporte de las necesidades grasas-, lo cual permite rebajar los aportes de hidratos de carbono y proteínas en forma de soluciones de baja osmolaridad.

\section{Bases fisiológicas}

Cuando se indica una alimentación parenteral en un niño es necesario tener presentes los siguientes puntos para programar las soluciones a administrar:

3.1. Necesidades calóricas: El consumo calórico para mantener un estado anabólico en un niño en crecimiento alimentado por vía oral, normalmente debe cubrir los requerimientos correspondientes a metabolismo basal, actividad física, crecimiento, acción dinámico-específica de los alimentos y pérdidas por deposiciones y orina. La necesidad diaria de calorias para las diferentes edades se señala en el cuadro $1.14,201$,

\section{Cuadro 1}

$\begin{array}{cr}\text { Peso (kq.) } & \text { Calorias metab. } \\ \text { RN } & 47+4 \\ 3-10 & 70+5 \\ 10-15 & 57+4 \\ 15-25 & 45+4 \\ 25-35 & 37+3 \\ 35-60 & 32+3 \\ \text { 8obre } 60 & 27+3\end{array}$

Al aportar los nutrientes por vía parenteral, se ahorran las calorías correspondientes a los mecanismos de digestión y absorción intestinal. Igualmente. debido al reposo obligado en el que están estos niños, el gasta calórico correspondiente a la actividad o ejercicio está muy dísminuido. Por todo ello, los aportes de $100 \mathrm{cal} . / \mathrm{kg}$./día se ha señalado como una cifra suficiente para el recién nacido y el lactante. ${ }^{121}, 22,23,24,251$

En el R.N. y prematuro se acentúa al máximo el problema del aporte energético por kg. de peso corporal, por ser éste muy elevado a esta edad, coincidiendo con escasas reservas calóricas, ${ }^{(22)}$ y estar limitada la tolerancia de grandes aportes hidrosalinos intravenosos. ${ }^{191}$

En etapas posteriores existe una disminución de las necesidades referidas al patrón de peso corporal kg/día.

Por el contrario, es necesario tener también presente el incremento del consumo calórico que se produce en las situaciones de stress: postoperatorios 
de todo tipo, traumatismos, infección, quemaduras, etc., en los que para evitar un estado catabólico ${ }^{20}$ será preciso incrementar oportunamente el aporte energético.

3.2. Necesidades hidrosalinas: En el cuadro $2^{(9)}$ se señalan las necesidades hidrosalinas normales diarias por cada 100 calorias metabolizadas. Con el lamado aporte hidrosalino de "mantenimiento" se

\section{Cuadro 2}

$\bullet$

Agua Por c/100 cal. metaboliz.

Pérdidas insensibles

- Pulmonares 13

- Piel 30

Sudor (usual)

$30 \quad 43 \mathrm{ml}$.

Orina

$20 \mathrm{ml}$.

$87 \mathrm{ml}$.

Deposiciones

$0-10 \mathrm{ml}$.

Total

$150 \mathrm{ml}$.

Agua metabólica

$-10$

$140 \mathrm{ml}$.

Electrólitos

Sodio

Potasio

Cloro

$3 \mathrm{mEq}$.

$2-3 \mathrm{mEq}$.

$3 \mathrm{mEq}$.

(Modificado de (Winters))

cubren las necesidades de agua y electrólitos en base al concepto de calorias metabolizadas, de Darrow. En este concepto se representan las calorías que se consumen en el metabolismo basal, más las que se utilizarán en mantener las variaciones de la temperatura corporal, asi como una minina actividad física (niño en una cama con goteo), etc. Como vemos en el cuadro 2, por cada 100 calorías que se metabolizan se necesitan $140 \mathrm{ml}$. de agua. ${ }^{(14,20)}$

Como en la alimentación parenteral damos una cifra elevada de calorías, aproximadamente 100 $\mathrm{cal} . / \mathrm{kg}$./día, el aporte hídrico que se necesitará será de $140 \mathrm{ml} . / \mathrm{kg}$. /día. Esto viene matizado igualmente en función de la osmolaridad de los sueros y la via venosa que utilicemos. Los sueros de alta osmolaridad precisaron obligadamente una vena de grueso calibre y alto flujo del tipo de las venas cavas: alimentación parenteral central.9, 14, 11, 22, 13, 14. 15, 26, Por el contrario, si utilizamos sueros con osmolaridad inferior a 800-1.000 mOs/litro, podremos administrarios por una vena de pequeño calibre y bajo flujo: alimentación parenteral periférica. Para facilitar esta técnica se ha llegado a administrar de 200$250 \mathrm{ml} . / \mathrm{kg} . / \mathrm{dia}^{(8,20)}$ tras incrementos lentos y progresivos.

Los electrólitos deben suministrarse de acuerdo a las necesidades por $100 \mathrm{cal}$. metabolizadas. Se añadirán los correspondientes a pérdidas extraordinarias (diarrea, vómitos, fístulas gastrointestinales, etc. $)^{19 \mid}$ Los yalores promedios de pérdidas por via digestiva se exponen en el cuadro 3 . De cualquier manera, el aporte electrolítico no debe constituir una sobrecarga de solutos al riñón (véase más adelante) (4.1.).

Cuadro 3

Promedios de pérdidas por via digestiva (iones)

\begin{tabular}{|c|c|c|c|c|}
\hline & $\mathrm{Na}^{+}$ & & $\mathbf{K}^{+}$ & $\mathrm{Cl}^{-}$ \\
\hline Jugo gástrico & $60(9-116)$ & 9 & $(0-32)$ & $84(8-154)$ \\
\hline Intestino delgado & $111(82-148)$ & & $5(2-8)$ & $104(43-137)$ \\
\hline lleostomía (reciente) & $129(105-144)$ & 11 & $(6-29)$ & $116(90-136)$ \\
\hline Lleostomía (adoptada) & 46 & 3 & & 21 \\
\hline Bilis & $149(131-164)$ & 5 & $(3-12)$ & $101(89-108)$ \\
\hline Páncreas & $141(113-153)$ & & $5(3-7)$ & $77(54-95)$ \\
\hline
\end{tabular}

Reproducido de Cole, W. G., y Ramos, J. (Surgical Clin. North Ameriea, febrero 1962).

Igualmente, debe tenerse presente, en la alimentación parenteral, la administración de otros electrólitos, como son el $\mathrm{Ca}^{++}, \mathrm{Mg}^{++}$, fosfatog y sulfatos, que son importantes para las diversas funciones metabólicas. Su déficit se deja sentir tras la falta de aportes por un periodo prolongado, como 
se ha visto en la alimentación parenteral. ${ }^{(16,17,27,28)}$ Las necesidades calcuiadas para vía endovenosa son: Calcio $2-4 \mathrm{mEq} / \mathrm{kg}$. /día; Fösforo: $3-6 \mathrm{mEq} / \mathrm{kg}$.

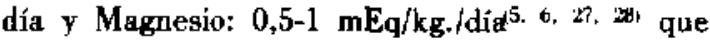
señala las necesidades basales y el aumento de los requerimientos en caso de pérdidas moderadas o excesivas.

También se debe tener presente el estado ácidobase del paciente. Alteraciones electrolíticas principalmente dependientes del $\mathrm{Cl}^{-}$y del $\mathrm{K}^{+}$pueden condicionar desajustes en el equilibrio ácido-base. Un control correcto de los aportes y las pérdidas previene esta problemática. Pero la complicación más frecuente - principalmente en los primeros años de utilización de la Alimentación ParenteraL fue la acidosis metabólica secundaria a un aporte excesivo de proteínas, bien debido a la canidad global de las mismas, ${ }^{(29)} 0$ bien a la influencia de ciertos aminoácidos ricos en enlaces $-\mathrm{SH}^{(3)} \mathrm{Ac}$ tualmente, con la reducción de los aportes proteicos no se plantea este problema.

3.3. Necesidades Energéticas (Principios Inmediatos): En los aportes energéticos por vía oral, el porcentaje de principios inmediatos en el mundo occidental oscila: $50 \%$ de hidratos de carbono, $35 \%$ de lípidos, $15 \%$ de proteinas.

En la Alimentación Parenteral, los porcentajes aproximados de los distintos principios inmediatos son aproximadamente: $70 \%$ de hidratos de carbono, $20 \%$ de grasas y $10 \%$ de proteínas.

La metabolización de los hidratos de carbono y de las grasas produce un aporte energético de 4 y 9 calorías por gramo, respectivamente. Como producto final de su metabolismo dan $\mathrm{CO}$ y $\mathbf{H}_{2} \mathrm{O}$. Las proteínas producen 4 calorias por gramo, y como principal metabolito terminal generan urea, que precisa una eliminación renal, hecho a tener presente en la valoración de la sobrecarga de solutos a este órgano durante la alimentación parenteral. ${ }^{\mid 3 t}$

En el cuadro IV se presentan en forma de gr./kg./día los aportes de los tres principios inmediatos y su repercusión energética en las dos técnicas de AP (Central y Periférica) en el niño. Estos valores serían válidos para una alimentación parenteral completa con los 3 principios inmediatos administrados diariamente. Vemos cómo existen variaciones er función del tipo de técnica utilizada: Centrało Periférica.

\section{Cuadro 4}

Aporte de los tres principios inmediatos en la AP en el niño

\begin{tabular}{lcccc} 
& \multicolumn{2}{c}{ AP Periférica } & \multicolumn{2}{c}{ AP Central } \\
& g./K/d. & Cal./K/d. & g./K/d. & Cal. $/ \mathrm{K} / \mathrm{d}$. \\
Hidratos de carbono & 18 & 72 & 24 & 96 \\
Proteínas (AA) & 2,5 & 11 & 2,5 & 11 \\
$\quad\left(N_{2}\right)$ & 0,3 & & 0,3 & 18 \\
Grasas & 2 & 18 & 2 & 18 \\
\hline Total & & 101 & & 125
\end{tabular}

\section{a) Los Hidratos de Carbono}

Los Hidratos de Carbono constituyen el principal aporte energético en la A.P. Existen diferentes tipos de Hidratos de Carbono para aplicación en A.P. El más recomendado es la glucosa. Constituye el monosacárido que utiliza el organismo más ampliamente. Tiene una metabolización inmediata por el sistema nervioso central. Como inconveniente precisa la acción de la insulina para su metabolización en muchos órganos, sin embargo, esto presenta una faceta positiva, al estimular el anabolismo corporal. ${ }^{\text {[32! }}$

Otro Hidrato de Carbono que también se ha utilizado es la fructosa. Presenta el inconveniente de su rápida metabolización con producción de ácido láctico (acidosis láctica).

Igualmente se han utilizado alcoholes como etanol, sorbitol, y xilitol. Su metabolización, aunque produce mayor aporte calórico que la fructosa: 7 calorías/gramo, tiene los mismos inconvenientes que los descritos en ésta, y no se recomienda su uso 
rutinario. Sin embargo, todos ellos pueden entrar en consideración en ciertas circunstancias. ${ }^{(33,34,35\}}$

\section{b) Los Lipidos}

Los Lípidos constituyen un segundo principio inmediato importante en la nutrición, de forma obligada, deben proyeer los ácidos grasos esenciales: ácídos linoleico, linolénico y araquidónico. Los diversos preparados utilizados están formados por una solución acuosa isotónica dé aceite de semilla de soja, emulsionado por lecitina de yema de buevo más un agregado de glicerol, xilitol o sorbitol, para proporcionar la isotonicidad. ${ }^{(5,2 \pi, 35,36,371}$

Los autores que no han empleado estos preparados han prevenido el déficit de ácidos grasos esenciales mediante tratsfusiones de plasma fresco, 1-2 veces por semana, ${ }^{(18,25,38)}$ si bien este proceder entraña alto riesgo infectivo (hepatitis) y elevado costo económico, lo que hace que no sea recomendable.

Debido a que se han descrito innumerables reacciones tóxicas por lipidos, se ha recomendado que la dosis diaria de éstos no sea superior a 2 gr. $/ \mathrm{kg}$. / día. El porcentaje de aporte calórico no debe superar el $30 \%$ del total. La duración del tratamiento no debe ser superior a 15 días seguidos. La velocidad de administración debe ser lenta ${ }^{17,}$, La "International society of Parenteral Nutrition" considera como requisitos de una emulaión lipídica los siguientes: debe ser libre de componentes lóxicos; el tamaño de las partículas no superior a una micra (Kilomicrones); no debe generar efectos secundarios; la temperatura del enfermo no debe elevarse más de $1^{\circ} \mathrm{C}$; al cabo de 24 horas de lipemia debe alcanzar valores normales. El único preparado que actualmente reúne estos requisitos y por tanto garantía - también avaladas por la experiencia-para su infusión es el INTRAL.IPID. ${ }^{6,34)}$ El Intralipid es una emulsión de aceite de soja, que tiene como emulsionantes lecitina de yema de huevo y glicerol. Existe en dos presentaciones al $10 \%$ y $20 \%$ (Cuadro 5).

\section{c) Las Proteínas}

Las Proteínas que se administren es importante que contengan todos los aminoácidos esenciales, asi como un porcentaje de AA no esenciales, que proporcionen una cantidad de $\mathrm{N}_{2}$ adecuada. Es importante considerar la cantidad de calorías que se administran por gramo de $\mathrm{N}_{2}$; el aprovechamiento máximo se obtiene cuando esta relación es de un gramo de $N_{2} / 150$ cal. o un gramo de $N_{2} / 200$ cal., para que no se utilicen los aminoácidos como fuente energética. ${ }^{(24}$. 39, 40) Durante la perfusión se deben

\section{Cuadro 5}

Composición cualitativa y cuantitativa de la emulsión grasa de aceite de soja (Intralipid)

(Tomado de la Casa Vitrum - Suecia)

$\begin{array}{ccc}\text { Composición } 1.000 & \text { Intralipid } & \text { Intralipid } \\ 10 \% & 20 \%\end{array}$

Aceite de semilla de soja $\quad 100 \mathrm{~g} . \quad 200 \mathrm{~g}$.

Lecitina de yema de huevo $12 \mathrm{~g}$. $12 \mathrm{~g}$. (Fosfátidos)

Glicerol

$25 \mathrm{~g} .25 \mathrm{~g}$.

Agua de inyección, C.S.p 1.000 c.c. 1.000 c.c.

suministrar alrededor de 0,02-0,03 gramos de $\mathrm{N}_{2}$ $\mathrm{kg}$ /hora; de eate modo el organismo aprovecha el $80-90 \%$ de los aminoácidos. ${ }^{39}$

Se debe considerar la cantidad y proporción de todos y cada uno de $\log \mathrm{AA}$. El total de AA esenciales $(E)$, debe tener una relación con el total de $\mathrm{N}_{2}$ que aportemos (T), (Relación E/T), superior a 3 . Las proteínas que tienen una relación $\mathrm{E} / \mathrm{T}$ superior a 3 se llaman de alto valor nutritivo.(41)

Por otra parte, se han estudiado aisladamente las cantidades de AA que se necesitan. Como fuente de información se han considerado las recomendaciones de Rose (1957), los datos de Hold-Snyderman, ${ }^{15.6,28\}}$ las necesidades de oposición de sustancias en el neonato nacido prematuramente..$^{(41)}$

Como proteínas patrón se han considerado la Proteína de Yema de Huevo y/o la leche de mujer, considerando que reúnes una composición idónea.

En el Cuadro VI exponemos la lista de los AA esenciales en todas las circunstancias, así como la de otros AA que por precisarge de forma obligada sólo en determinadas ocasiones se denominan semiesenciales. ${ }^{|6|}$

Los primeros preparados de aporte proteico fueron los hidrolizados de caseina o de fibrina, proteínas de alto valor proteico. Actualmente todos los preparados de hidrolizados lo son a expensas de caseína. La mayor ventaja que presentan es la baja asmolaridad de estas soluciones que permiten su utilización periférica. El mayor inconveniente es el tener una composición de $\mathbf{A A}$ cuantitativa y cualitativamente varigbles y desconocidas. Sin embargo, hoy día la tendencia más extendida es la de utilizar soluciones sintéticas de aminoácidos, en los cuales se aportan AA en cantidad y concentración conocida.

Las cantidades de aporte protejco recomendables en el recién nacido y en el niño pequeño son de 2,5 gramos/kg/dia, equivalentes a $330 \mathrm{mg} / \mathrm{kg} / \mathrm{día}$ de $\mathrm{N}_{2} .^{16,42)}$ 


\section{Cuadro 6}

AA esenciales y semiesenciales en el niño

Isoleucina

Leucina

Valina

Lisina

AA esenciales

Metionina

Fenil alanina

Treonina

Triptofano

Cișteina

A A esencial en el feto

Tirosina

A A esencial en el prematuro

Histidina

AA esencial en niños pequeños y en estado urémico

Arginina

AA esencial para mejor utilización de los otros $A$ A

(Modificado de A. Wretlind, Estocolmo.)

\subsection{Necesidades de Oligoelementos: Se han des-} crito 14 elementos minerales esenciales que se necesitan en cantidades mínimas: Hierro, iodo, zinc, cobre, cobalto, molibdeno, selenio, manganeso, cromo, níquel, vanadio, estaño y flúor. Respecto a sus funciones metabólicas, forman parte de metaloenzimas, o tienen una función estructural, formando parte de ácidos nucleicos, porfirinas, hormonas, vitamina $B_{12}$, etc. Se conocen las necesidades por vía oral, de sólo algunos de ellos: hierro, yodo, zinc, cobre, manganeso, cromo y flúor. $\left.{ }^{(5,} 16,17\right)$ En el Cuadro 7 se exponen las necesidades recomendadas para la vía intravenosa. ${ }^{27}$

\section{Cuadro 7}

\section{Necesidades RN y lactante ( $\mathrm{M} \mathrm{mol} / \mathrm{kg}$./dia)}

\section{Necesidades basales Pérdidas + NB}

$\begin{array}{llc}\text { Hierro } & \mathbf{2 , 0} & \mathbf{3 , 0}-\mathbf{4 , 0} \\ \text { Yodo } & 0,04 & 0,1 \\ \text { Zinc } & 0,6 & 1,5 \\ \text { Cobre } & 0,3 & - \\ \text { Manganeso } & 0,3 & 1,0 \\ \text { Molibdeno } & 2,0-4,0 & \mathbf{4 , 0}-\mathbf{6 , 0} \\ \text { Cromo } & 0,01 & - \\ \text { Fluor } & 3,0 & -\end{array}$

Se han descrito signos de déficit de oligoelementos en la desnutrición calórica-proteica, en die- tas elementales, en prematuros y en la alimentación parenteral total. En esta última se han encontrado bajos niveles séricos de cobre y zinc, en alimentación parenteral de alrededor de 60 días; $;^{(5)}$ de manera que serían los elementos que mayor importancia tienen en ser administrados durante la alimentación parenteral completa.

Los oligoelementos se pueden administras mediante soluciones preparadas con esta finalidad ${ }^{(6)}$ aunque otros autores al igual que con los ácidos grasos esenciales los proporcionan mediante transfusiones de plasma o sangre fresca, 1 ó 2 veces por semana. ${ }^{18,20,33)}$ Actualmente este proceder es rechazable, por los riesgos de hepatitis que representan las transfusiones múltiples y la carestía que suponen.

3.5. Necesidades de vitaminas: En alimentación parenteral hay pacientes que han presentado deficiencias de vitaminas hidrosolubles al cabo de $2-3$

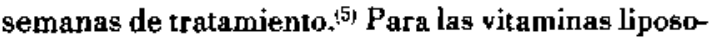
lubles el plazo es aigo mayor. ${ }^{|5|}$

Las necesidades orales son bien conocidas. Para la via venosa se han hecho las siguientes recomendaciones (Cuadro 8 ). ${ }^{273}$

Las vitaminas se administran en la solución parenteral mediante preparados comerciales que llevan las hidrosolubles y las liposolubles conjuntamente. Aquellas que no estén contenidas en el preparado I.V., como la Vitamina K, el ácido fólico y la cianocobalamina, deben administrarse por vía I.M. a intervalos regulares de tiempo. Deben proporcionársele al paciente desde el comienzo de la alimentación parenteral. ${ }^{(5)}$

\section{Peculiaridades Metabolicas a considerar en la Alimentación Parenteral.}

4.1. Sobrecarga de Solutos al Riñon: En la alimentación parenteral, así como en la oral, debe cansiderarse este concepto fisiológico a fin de no sobrecargar el rinón del R.N. y lactante pequeño que concentra la orina hasta unos $600-700 \mathrm{mO} / \mathrm{litro}$, bo cual significa, que una oferta mayor de solutos al sobrepasar el tope de concentración de la orina eliminará los solutos a expensas de una diusesis osmótica, lo cual produce deshidratación generalmente de tipo hipertónico. Los solutos que tienen eliminación preferentemente renal ${ }^{31)}$ son la urea y ciertos electrólitos. De acuerdo al concepto de Fomon, ${ }^{(31)}$ es posible calcular la sobrecarga total de solutos al riñón en base a las proteinas y electrolitos proporcionados por la dieta o la solución de alimentación parenteral, sabiendo que 1 gramo de protejna proporciona $4 \mathrm{mOs}$ en forma de urea y que cada 
Cuadro 8

Necesidades RN y lactante ( $\mathrm{K}_{\mathbf{g}} /$ /día)

Necesidades basales

Pérdida + NB

Tiamina

Riboflatina

Nicotinamida

Piridotina

Acido folico

Cianocobalamina

Acido pantoténico

Biotina

Acido ascórbico

Retinol

Ergocalciferolo

Colecalciferol

Fitilmenaquinona

Tocoferol

\begin{tabular}{|c|c|}
\hline & $\begin{array}{l}5 \mathrm{mgr} . \\
8 \mathrm{mgr} .\end{array}$ \\
\hline 1,0 & mgr. \\
\hline 0,1 & mgr. \\
\hline 20 & $\mathrm{~m}=\mathrm{g}$. \\
\hline $\begin{array}{l}0,2 \\
1,0\end{array}$ & mcg. \\
\hline $\begin{array}{c}1,0 \\
30\end{array}$ & mgr. \\
\hline $\begin{array}{l}30 \\
3,0\end{array}$ & meg. \\
\hline $\begin{array}{l}3,0 \\
0,1\end{array}$ & mgr. \\
\hline 0,1 & mgr. (333 UI) \\
\hline & mgr. (100 UI) \\
\hline & mgr. \\
\hline
\end{tabular}

$\begin{array}{ccl}0,1 & -2,1 & \text { mgr. } \\ 0,2 & -0,4 & \text { mgr. } \\ 2,0 & -4,0 & \text { mgr. } \\ 0,2 & 0,6 & \text { mgr. } \\ 40-50 & \text { mcg. } \\ 0,4- & 5,0 & \text { mcg. } \\ 2,0- & 2,0 & \text { mgr. } \\ 60-60 & \text { mcg. } \\ 6-20 & \text { mgr. } \\ 0,1- & 0,15 & (333-500 \text { UI }) \\ 2,5- & 2,5 \text { (100 UI) } \\ 50-150 & \\ 1,0- & 1,5 \text { UI }\end{array}$

0,1 - 2,1 mgr.

2,0 - 4,0 mgr.

0,2 - $0,6 \mathrm{mgr}$.

40 - 50 mcg.

0,4 - 5,0 mcg.

2,0 - 2,0 mgr.

$60-60$ meg.

6 - 20 mgr. miliequivalente de $\mathrm{Na}, \mathrm{K}$ y $\mathrm{Cl}$ producen un miliosmol más..$^{(31)}$ Según la fórmula:

SRTS mOs $=$ (g. proteinas $\times 4)+(m E q \mathrm{Na}+\mathrm{K}$
+ Cl) (l)

en ella no se suman los restantes electrólitos y oliguelementos, porque se hace una composición con los $\mathrm{mEq}$ de $\mathrm{Na}, \mathrm{K}$ y $\mathrm{Cl}$ proporcionados que normalmente se retienen por el organismo.

Es posible también, conociendo la SRTS, calcular la osmolaridad urinaria en base a la siguiente fórmula:

$$
\mathrm{O}_{2}=\frac{\mathrm{S}_{\mathrm{l}}-\mathrm{Scr}}{\mathrm{H}_{2} \mathrm{O}_{1}-\mathrm{H}_{2} \mathrm{O} \text { P.i. }}
$$

$\mathrm{O}_{\mathbf{u}}=$ Concentración Urinaria

$S_{\gamma}=$ Solutos totales de dieta o parenteral (Ver fórmula li

Scr $=$ Solutos del crecimiento. Por cada gramo de peso que aumente el crecimiento diario se retienen $0,9 \mathrm{mOs}$ de solutos $(0,9 \times$ gramo $d e$ peso que aumente el crecimiento diario).

$\mathrm{H}_{2} \mathrm{O}_{1}=$ Agua total (líquidos aportados)

$\mathrm{H}_{2} \mathrm{O}_{\mathrm{r}, \mathrm{i} .}=$ Agua de la Perspiración insensible. (30)-

$50 \mathrm{ml} / \mathrm{kg} / \mathrm{dia}$ ).

El agua de oxidaciôn y la de las heces se equilibran mutuamente. Ello puede valernos para garantizar la corrección de los aportes I.V. que estamos programando a un sujeto determinado.

4.2. Osmolaridad de la solucion empleada: Concepto que interesa principalmente para la elección de la vía de administración de la solución: vía central o periférica. En el caso de una solución con una osmolaridad superior a $1.000 \mathrm{mOs} /$ litro se debe usar obligadamente una vena central (vena cava superior o aurícula derecha), que al tener gran flujo permite una adecuada disolución.

La osmolaridad de la solución se calcula sumando los aportes en $\mathrm{mOs} / \mathrm{kg} /$ dia proporcionados por: la glucosa $(5,5 \mathrm{mos}$ por gramo de glucosa), es decir: $\mathrm{g} / \mathrm{kg} /$ dia $\times 5,5$; proteínas $(\mathrm{mOs}$ por gramo de AA), g $/ \mathrm{kg} / \mathrm{dia} \times 11$, y los mEq/kg/dia de cada uno de los iones, relacionándolo con el líquido aportado por $\mathrm{kg} / \mathrm{dia}$; mediante regla de tres se calcula la osmolaridad por litro. ${ }^{24)}$

\section{Preparación de la solución para Alimentacion Parenteral.}

Las necesidades diarias indicadas para los diversos principios, pasan a ser responsabilidad del farmacéutico para la fabricación de la solución. (Véase 2.)

La preparación y uso de la solución debe cumplir con los siguientes requisitos: a) debe estar exenta de gérmenes y pirógenos; b) no debe contener productos precipitados; c) debe mantenerse refrigerado a temperatura de $+4^{\circ} \mathrm{C}$ antes de su utilización para evitar la proliferación bacteriana y de Candidas Albicans; ya que se ba demostrado la proliferación de estos hongos a las 24 horas de mantenerse una solución proteica a la temperatura ambiente, $\left.{ }^{(431} d\right)$ se ha recomendado el fraccionamiento en 2 frascos, de las necesidades para 24 horas, a fin de evitar este último inconveniente. ${ }^{.431}$ 
6. Técnicas y Vías de Administración de las Soluciones para Alimentación Parenteral.

El volumen de solución calculado para las 24 horas debe ser administrado a une velocidad constante, para lo cual es preciso se perfunda mediante bomba de infusion $I V$, independiente de la via venosa que utilicemos. Cuando los líquidos a perfundir tengan una alta osmolaridad (altos aportes energéticos disueltos en escasa cantidad de agua) tendremos que utilizar una vena de gran calibre, y por el contrario, si el volumen de líquidus IV es grande, la usmolaridad de la solución se reduce y podremos valernos de venas periféricas.

6.1. Via Central: Ideada por Dudrick y col. en $1967{ }^{\left(y_{1}\right)}$ permite la administración de soluciones con elevada osmolaridad, mediante un catéter en vena cava superior o aurícula derecha. Debido a que no se usan lípidos, se ha tenido que recurrir a un aumento de la concentración de los hidratos de carbono como fuente energética, lo cual eleva considerablemente la osmolaridad $44,45,46,47,46,44,50,51,52.53,54,531)$

De este modo se llega a proporcionar hasta 24 $\mathrm{g} / \mathrm{kg} /$ día. Esta cifra se debe alcanzar de forma progresiva. Partiendo de $12 \mathrm{~g} / \mathrm{kg} / \mathrm{dia}$, se aumentarán 1-2 g/kg/día cada nuevo dia.|2il| Aproximadamente en 6-7 días se alcanzará el tope de aportes. Las necesidades del resto de los nutrientes (proteínas, oligoelementos y vitaminas) son las ya descritas, que se mantendrán er los valores recomendados desde el $2{ }^{\circ}-3 .^{\circ}$ día de parenteral.

ASPECTOS TECNICOS: La colocación de la terminaciún de un catéter en la auricula derecha o en vena cava superior, se realizará desde: a) Venotomía de yugular externa o interna, en la unión del 1/3 medio con el $1 / 3$ inferior del cuello con creación de un túnel subcutáneo. b) Venotomia de vena basûlica, introduciendo catéter hasta aurícula derecha o vena cava superior. Creación de tunel subcutáneo.

Cateterización de la vena: Se introduce catéter de plástico que sepamos que se tolera largo tiempo por el organismo (polipropileno, polietileno, cloruro de polivinilo, etc.) según las técnicas clásicas de venatomia. Se avanza el catéter hasta ubicarto en el lugar deseado. Se realiza comprobación radiológica (catéteres con línea radioopaca o rellenados de contraste radioopacu). Se fija la salida del catéter a piel de forma segura. En el punto de salida se coloca un apósito con Betadine (antibacteriano y fungicida). El catéter se conecta a un sistema de goteo con un filtro Millipore de 0,22 mictas. ${ }^{(5,4)} \mathrm{Se}$ completa el circuito con el frasco o bolsa con los nutrientes, y una bomba de infusión (Esquema I). Por tanto, esta técnica requiere:

\section{ESQUEMA I:}

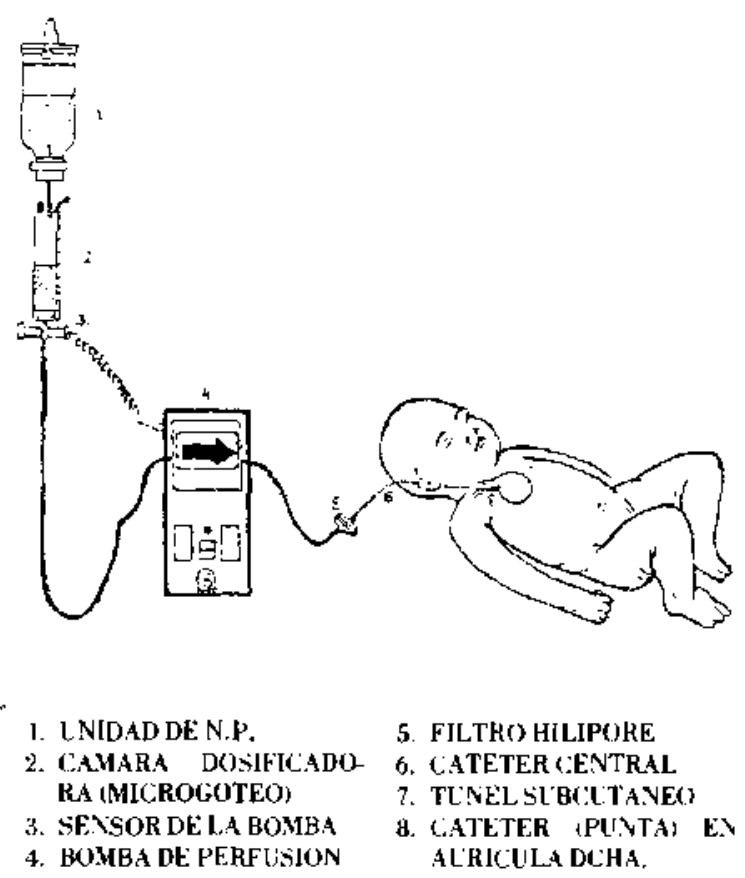

Esquema del montaje de una Alimentación Parenteral Central en el niño.

a) Cuidados de asepsia máxima a fin de reducir los riesgos de infección. (10)

b) Como hemos dicho, la solución debe pasar a través de un filtro antibacteriano Millipore de 0,22 micras, que debe cambiarse cada dia. Cambio de apósitos y colocar betadine en el sitio de entrada del catéter diario. ${ }^{3,9}, 44, ; 6$,

c) Bomba de infusión, que permite su administración a una velocidad continua. ${ }^{(5,41}$

d) Administración de lípidos por to menos 2 veces por semana. ${ }^{(9)}$ Lo correcto es la administración diaria de lípidos. No parece aconsejable el método de aportar ácidos grasos y/o oligoelementos mediante transfusiones semanales de plasma, tanto por el aporte insuficiente e incorrecto de las mismas, como por el elevado costo económico y riesgo de contagio de hepatitis.

6.2. Via periférica: Permite la administración de soluciones con una osmolaridad alrededor de 600 $800 \mathrm{mOs} /$ litro, a través de cualquier vena periférica. ${ }^{\{2\}, 24,57,58,59\}}$

Esta vía permite la administración de todas las 
necesidades nutriemtes, tan súlo con un aumento de las necesidades hídricas a $166 \mathrm{ml} . / \mathrm{kg}$. $/$ día, manteniendo el aporte calórico en $101 \mathrm{cal} / / \mathrm{kg} . / \mathrm{dia} .^{(2 \mathrm{~d})}$

Los lípidos se administran en un frasco separado por un tubo en $Y$ conectado al sistema de infusión, dado que las particulas grasas no atraviesan el filtro de 0,22 micras del sistema. Otros autores, que no usan lipidos, han administrado soluciones que proporcionan los requerimientos de los hidratos de carbono, hasta $32 \mathrm{gr} / \mathrm{kg} . / \mathrm{día}{ }^{(18)} \mathrm{La}$ disminución de la osmolaridad de la solución la han conseguido en base a un aumento considerable del aporte hídrico a $200-250 \mathrm{ml} / \mathrm{kg} . / \mathrm{dia}$, lo que debe lograrse en forma progresiva.

El resto de los requerimientos son los señalados.

La técnica consiste en: Punción venosa con una aguja Butterfly N." 25 . Se mantiene lo máximo que aguante en cada vena. Requiere de los mismos cuidados de asepsia indicados para la vía central. En lo posible debe usarse una bomba de infusión. Deben administrarse lípidos 2 veces por semana. ${ }^{[4,39]}$

\section{Controles a realizar durante lo Alimetlación Parenteral.}

Estando hoy día demostrado a niveles clínicos, metabólico y experimental, la eficacia de la alimentación parenteral, $\left.{ }^{(5,}, 0,4+21,24\right)$ parece innecesario establecer controles muy rigurosos a tales niveles en cada paciente. Por tanto, nuestra tendencia actual es establecer controles que sean eminentemente prácticos y de base clínica, que nos permitan conocer la respuesta de cada paciente a esta técnica. Resumiremos, sin embargo, una lista de las posibilidades de control durante la alimentación parenteral.

\subsection{Control de consiantes fisiológicas: Tempera-} tura, Pulso, Respiración y, presión arterial, se puede controlar cada 6-8-12 horas, "ati" según la siluación clínica de cada paciente.

\subsection{Control de crecimiento Pondo-estaturals:}

a) peso, de riguroso control diario.

b) talla y perímetro craneal, su control será semanal o cada 15 dias.

7.3. Controles metabólicos. ${ }^{4}$. 10. 14. 20. 35, 33, 45. 16. 5.5. 60, 61, 62)

a) Agua: Mediante BALANCE HIDRICO: entradas y salidas: Se realizará diariamente.

b) Electrolitos ( $\mathrm{Na}, \mathrm{Cl}$ y $\mathrm{K}$ ): séricos y urinarios, se analizarán durante los primeros días de AP y posteriormente con una secuencia semanal. c) Osmoloridad en sangre y orina y relación osmolaridad urinaria/osmolaridad plasmática $(0 \mathbf{0} /$ Opl para estudiar el estado hidrosalino y la función renal. Control diario durante los primeros dias y posteriormente semanal.

d) Hidratos de carbono: Determinación de glicemia por micrométodo (Labstix) y glucosuria (Dextrostix), cada 8 horas. De esta forma podremos alcanzar el valor máximo de aporte IV de ghucosa, que no produzca glucosuria.

e) Proteinas: Balance Nitrogenada de ingresos y salidas, siempre que queramos diferenciar si el aumento de peso se debe a retención de agua (edemas) o es un auténtico incremento ponderal (aumento de masa corporal).

Otros análisis pueden ser:

-Determinación semanal de BUN o Urea, y Creatinina en sangre.

-Determinación de $\mathrm{N}_{2}$ total (Urea, Creatinina y $\mathrm{NH}_{3}$ ) en orina. Con frecuencia variable, hasta Llegar al valor máximo de administraciún de pruteínas: a continuación con una secuencia semanal o quincenal.

-Análisis de Aminoácidos en Sangre y Orina: potestativo o con una frecuencia variable en casos muy especiales.

-Determinación de proteínas en sangre y relación Albúmina/Globulina, inicialmente cada quince días.

- Determinación de pH y gases en sangre, cada 2-3 días, debido a que se ha producido acidosis metabrulica para algunas soluciones proteicas. ${ }^{12}, 301$

f) Lipidos: Determinación de lipemia y colesterolemia de forma variable hasta llegar al valor má ximo de administración de lípidos; luego semanal.

- Determinación cromatográfica de ácidos grasos, para conocer sus niveles plasmáticos y/o valorar alteraciones de su metabolismo.

g) Metabolismo Fosfo-Calcio: Calejo, Fésforo $\mathrm{y}$ fosfatasas alcalinas semanal o quincenal.

h) Oligoelementos ( $\mathrm{Zn}$ y Cu) y otros metales, si se desean investigar sus deficiencias.

\subsection{Hematológicos:}

a) Hemoglobina, hematocrito y leucocitos cada 2-3 días al inicio, posteriormente de forma semanal. b) Coagulaciốn: De acuerdo a la clínica.

\subsection{Funcion Hepática:}
a) Transaminasas
b) Bilirrubina
c) Tiempo de protrombina
quincenal, pu- diendo reali- zarse semanal, según evolu- ción clínica y analitica.

\subsection{Bacteriología, Hongos:}

a) Hemocultivos para Bacterias y Hongos. 
b) Urocultivos (Candida Albicans).

c) Otros cultivos bacteriológicos (Frotis faríngeo y nasal, Frotis umbilical, coprocultivo, ete.).

Se solicitan ante la primera sospecha de cuadro infeccioso.

\subsection{Radiografia de Tórax: Tras inserción del caté-} ter para ver ubicación del mismo en la técnica central. Ante patología pulmonar sobreañadida, suspecha de movilización del cutéter central o alguna otra circunstancia clínica que la aconseje.

\section{Manejo de la técnica de alimentación pa- renteral.}

8.1. Referente a las soluciones: Las soluciones procedentes de farmacia deben ser manipuladas con esmero. Hasta su puesta al paciente deben conservarse en nevera a temperaturas entre $4-12^{\circ} \mathrm{C}$. No se utilizarán como vehículo para aportar medicamentos; ni mediante tubo en $Y$, simultáneamente con transfusiones, etc. Antes de su inmediata puesta al paciente, se mantendrán a temperatura ambiente, para que de nuevo adquieran temperatura normal. Se observará si tienen en suspensión alguna(s) particula(s), si está turbio o si ha variado de color (si adquiere color marrón).

\subsection{Referente a montaje del catéter y sistemas:} Se realizará con estricta asepsia. tal como ya ha sido comentado. Se controlará radiológicamente la gituación de la punta terminal del catéter. Se conectará el catéter al resto del sistema, con bomba incluida, montados igualmente con técnica estéril. (44, (3)!

Una vez fijado el catéter en su posición currecta, se cubrírá su salida con apósitos que se cambiarán, extremando la asepsia, cada $24-48$ horas ${ }^{(5,44,63)}$

8.3. Referente a la programación: Es muy importante el mantenimiento de una cifra constante de infusión, mediante bomba de infusión que impulga la solución a través del filtro del sistema, a la vez que garantiza un flujo constante. Si en algún momento se produjese algún desfase entre el volumen perfundido y el programado, no recuperar bruscamente esta diferencia, pues provocaríamos una subrecarga, con el riesgo subsiguiente de diuresis osmótica.

Si bruscamente apareciese fiebre de causa no filiada o justificada, se practicará un chequeo bacteriológico (hemocultivo, urocultivo, coprocultivo, etc.), con cultivo del líquido de NP, y se instaurará tratamiento antihiótico. 44 ' Deben vigilarse de forma especial las Candidas. Una vez conocido el gennen, se adaptará convenientemente el tratamiento antinfeccioso. Si no responde a éste, se debe retirar el catéter, pasando la NP a la vía periférica. Igualmente podrá adaptarse la NP a la situación infectiva. Una vez superada la fase de la infección, se podrá reinstaurar la técnica central.

\section{Indicaciones de la alimentación parenteral.}

La indicación esencial o más importante la constituye la imposibilidad o dificuitad de proporcionar una alimentación oral (ya sea por boca, sondaje o gastrostomia), (j) por un espacio prolongado de tiempo, en el que se desarrollará un estado catabólico. Así, se ha descrito su uso en afecciones gastrointestinales congénitas, $\left.{ }^{, 3}, 11.18,35,34,16,51\right)$ (atresja esufágica con fístula traqueoesofágica, obstrucciones intestinales, etc.) y adquiridas ${ }^{2}, 3,11,13,18.61$ (pancreatitis, fístulas de intestino delgado, obstrucción intestinal, etc.), en espera de proceder a su corrección quirúrgica. Otra indicación es cuando se precise reposo del tubo digestivo; de este modo se ha empleado en la anterocolitis necrotizante. ${ }^{[18,52\}}$ diarrea crónica inespecifica, ${ }^{(33,35,38.511}$ afecciones metabólicas congénitas, fístulas de intestino delgado, etcétera. ${ }^{\text {(tat, 65: }}$ Aunque su empleo preferente se ha señalado en patologia gastrointestinal, hay otros cuadros con aumentu signiticativo de las necesidades calóricas de Nitrógeno y/o de otros nutrientes, siendo insuficiente la vía oral. ${ }^{651}$ Así se ha indicado en casos de prematuridad extrema, desnutrición grave, quemaduras extensas, ${ }^{(5 .}$. I') pacientes intensivos en estado hipercatabólico, ${ }^{(24,36,61)}$ etc.

Por último, algunos autores han tratado con éxito la uremia e hiperkalemia de la insuficiencia renal aguda con infusiones intravenosas de glucosa y aminoácidos, reduciendo la necesidad de la diálsis. ${ }^{14}, 28.61$,

\section{Complicaciones de la alimentación paren- teral.}

10.1. Derivadas de la técnica empleada: En la aljmentación parenteral periférica no se han descrito complicaciones con un manejo adecuado. En la aljmentación parenteral central se han descrito las siguientes complicaciones:

10.1.1. Perforación de vena cava superior.

10.1.2. Embolia aérea. ${ }^{1601}$

10.1.3. Neumotorax $\left.{ }^{4,}{ }^{5} ; 1\right)$ con cateterización a través de función percutánea de vena subclavia.

10.1.4. Mala ubicación y desplazamiento de] catéter, lo que permite la trombosis al quedar localizada en una vena de menor calibre..$^{[33,6)}$

10.1.5. Trombosis venoga con tumefacciôn del territorio venoso correspondiente. ${ }^{\text {(14. } 53.66 !}$ 
Se ba descrito como más peligrosa en casos en que se ha cateterizado la vena cava inferior. La trombosis venosa obliga a la retirada del catéter. Se han descrito algunos casos de embolia, pero sin mayores peligros.

10.1.6. Infección: a) Local, ${ }^{(67)}$ en el sítio de entrada del catéter en la piel. b) General: septicemia, $\{3,12,3 \overline{3}, 38,40, \hat{2} 2,33,63,60,67,68\}$ ya sea por bacterias $\operatorname{gram}^{(+)}$y gramt $^{-1}$ y Candida albicans, favorecida por la desnutrición y por irritación de las paredes venosas por suluciones hiperosmolares en el sitio de implantación del catéter. Se han descrito cifras variables de septicemia: $2 \% d^{66}$ en adultos y $8 \% d^{35}$ a $16 \%^{38}$ en nin̄os. Se han encontrado cultivos positivos de catéteres en el $5,2 \% \%^{(27)}-5,9 \% \alpha^{(4)}$ de los casos.

\subsection{Metabolicas:}

\section{2. . Por hidratos de carbono:}

a) glucosa:

- Hiperglicemia. Glucosuria, que por diuresis osmótica puede producir deshidratación y coma hiperusmótico no cetónico. ${ }^{(4,35,52.53,55,70)}$ Por este motivo, una solución hiperosmolar en base a la glucosa debe ser administrada mediante una bomba de intusión a una velocidad continua de $0,75-1 \mathrm{~g}$ de glucosa/kg/hora.

- Hipoglucemia postinfusión. ${ }^{(35.46,55,70)} \mathrm{La}$ suspensión básica de la administración puede producir esta complicación debido a los elevados niveles de insulina que se han encontrado.

- Pérdida de iones, por diuresis usmótica.

b) Fructosa: Cuando ha sido empleada, debido a que su nivel de excreción renal es menor que el de la glucosa, ha tenido que ser infundida a una velocidad de $0,3 \mathrm{~g} / \mathrm{kg} / \mathrm{hora}$. Se ha descrito acidosis metabólica e hipoglucemia, entre otras complicaciones que produce.

\subsubsection{Por soluciones de proteinas:}

a) Acidosis metabólica, ${ }^{12 y}$, 30.401 derivada de los radicales $\mathrm{SH}^{-}$de las AA azufrados, del aporte amoniacal, pero esencialmente del $\mathrm{Cl}^{-}$y del $\mathrm{HCl}$ de la solución de AA cristalinos (acidosis metabólica hiperclorémica). ${ }^{\text {(70 }}$

b) Hiperamoniemia: Derivada del excesivo amonio en las soluciones de hidrolizados de proteinas, ${ }^{100}{ }^{71}, 721$ deficiencia de arginina, ornitina, ácido aspártico y ácido glutámico en las soluciones de aminoácidos cristalinos; por defecto hepático primario. El $\mathrm{NH}_{3}$ se metaboliza transformándose en urea y tambièn se transforma en $\mathrm{ClNH}_{4}$ en el riñón; es importante considerar la sobrecarga que presenta para el riñón del RN y lactante menor.

c) Azoemia prerrenal: Por infusión excesiva de hidrolizado de proteinas o aminoácidos. ${ }^{(2)}$ d) Disbalance de aminoácidos: ${ }^{(i)}$ Aminoacidurias. Por el uso de una solución de nutrientes con proporción no fisiológica de aminoácidos. Si la aminoaciduria es muy intensa, puede producir diuresis osmótica.

e) Disbalance iónico, por algunos iones contenidos en las suluciones proteicas. Así un exceso de Sodio puede producir edemas y un excesu de Cloro, acidosia metabólica hiperclorémica.

El uso de soluciones proteicas esta contraindicado en el coma hepático.

\subsubsection{Por emulsiones grasas:}

a) Reacción precoz o coloidal. ${ }^{19}, 36,37,3 \%$. 4ansiste en enrojecimiento, escalofrios, fiebre, taquipned, náuseas, vómitos, cefalea, etc. Ha sido interpretada como producida por pirógenos o reacción alérgica a una sustancin extraña. Se ha observado en el $1,6 \%$ de los casos. ${ }^{(34)}$

b) Reacción tardia: ${ }^{|H+36, k 3,34.46\rangle}$ Se han observado inicialmente después del uso repetido de infusiones de lípidos. Consisten en el "sindrome de sobrecarga", que se manifiesta por:

- Hepatoesplenomegajia, ${ }^{(8)}$ por depúsito de lipidos en las células del SRE. Lo mismo se ha visto en el tejido pulmonar. ${ }^{55,28,36,37 \text { ? }}$

- Hiperlipemias, con curvas de desaparición sanguínea más lentas luego de varios dias de administración. La hiperlipemia desaparece con heparina, al estimular ésta la actividad de la "lipasa lipoproteica" del plasma.

- Alteración de la coagulación sanguínea: Se ha observado un aumento de ésta provocando hemorragia $^{\text {tht }}$ y plaquetopenias.

- Ictericia! 5 , zot

- Anemia hemolítica. BSP. ${ }^{\text {(55) }}$

- Elevación de las transaminasas y del test

- Elevación de las proteínas plasmáticas y de la relación albúmina/globulina.

- Aparición de pigmento en el SRE. ${ }^{\text {(B) }}$

Despues de haber sido retiradas las primitivas soluciones lipídicas a causa de sus complicaciones, se han hecho estudios a fin de obtener un producto lo más parecido a los quilomicrones del plasma, así con el Intralipid (aceite de semilla de soja emulsionado con fosfolipidos de huevo) se han reducido considerablemente las complicaciones, siendo aceptado su uso primero por la escuela europea, y desde 1978 por la nurteamericana.

c) Déficit sérico de ácidos grasos esenciales, ácido linoleico y ácido araquidónico, con aparición de una dermatitis. Se ha observado en casos de uso de soluciones parenterales son inadecuada administración de ácidos grasos esenciales. ${ }^{355}, 701$

El uso de lipidos está contraindicado en: Hepa- 
to-esplenopatias graves. Reticulohemoblastosis. Hiperlivemias, Síndrume nefróticu, Shock Diátesis hemorrágica. Trombosis. Tromboflebitis. Embarazo. ${ }^{(34)}$

\subsubsection{Por aportes hidrosalinos:}

a) Disbalances hídricos: Edema, Deshidratación, Edema pulmonar aguda. ${ }^{(1)}$

b) Disbalances electrolíticos:

- Derivados de diuresis osmótica, por una alta sobrecarga osmótica o de aportes hidricos y salinos excesivos a insuficientes. Los prototipos metabólicos por déficit más observados son: Hiponatremia, Hipocloremia, Hipopotasemia, ${ }^{152}$. 34 " Hipocalcemia, ${ }^{(35}, 201$ Hipofosfatemia, ${ }^{(35,}{ }^{20)}$ Hipomagnesiemia. ${ }^{(70)}$

Los derivados de un aumento de los aportes: Hipercalcemia, ${ }^{(7))}$ Hiperkalemia, ${ }^{(70)}$ Hipernatremia, etcétera.

10.2.5. Por oligoelementos: Alteraciones provocadas por déficil de:

a) Zinc: Anorexia, retardo del crecimiento.

b) Cobre: Anemia, leucopenia, anormalidades óseas (radiológicas), degeneraciones del SNC.

c) Hierro: Anemia.

d) Cromo: Test tolerancia a la glucosa anormal.

e) Iodo: Hipotiroidismo.

10.2.6. Por vitaminas: Las deficiencias de cada una nos dará signos específicos.

\subsubsection{Otras:}

a) Anemia. Por deficit de hierro, cobre, ácido fólicod ${ }^{(2) !}$ o vitamina $B_{12}$.

b) Hemorragia. ${ }^{(70)}$ Por déficit de vitamina $K$.

c) Elevación de SGOT y de SGPT y de fosfatasas alcalinas séricas. Por inducción de enzimas secundariag ${ }^{702}$ a metabolismo acelerado de la glucosa; posible hepatotoxicidad secundaria a imbalance de AA; excesivo depósito de glucógeno y grasas en el hígado.

Prácticamente todas o casi todas estas complicaciones son evitables programando y controlando adecuadamente la N.P. Cuanto menor número de complicaciones tengarnos, mayor y más equilibrado sea el aporte de nutrientes, mejor serán los resultados que obtendremos. La N.P. completa es una técnica que ya ha demostrado su eficacia en el niño; la existencia de estas complicaciones evitables no debe inhibirnos de su utilización.

\section{REFERENCIAS}

1 Peoston, W. J Alimentación intravenusa. The Practilioner. 106: 68-78, 1974.

2 Dudrick, S. J, y Rhoads, J. E. Vew horizons for intravenuus feeding. JAML 215: 939-949, 1971.

3 Johnston, J. Parenteral teedung. The Practitioner 206: 103-110. 1971.

4 Orving, I, M., y Bomar, W. E. Parenteral Hiperalimentation and its practical applications. Anals. of Surgery 175: 712-719. 1972.

5 Ghudimi, H. Tutal Parenteral Nutrition. Premises and promises. Edited by H. Gluadimi, Juhn W. Illey and sons. New York. 1975.

6 Wretiind, A. Complete intravenuus nutrition. Theorelical and experimentel background. Nutr. Metabol. Suppl. 14: 1-j7, 1972.

7 Duchesne, G. L'Alimentation Parénlèrale for émulsions lipidiques, La Revue de Practicien. 24: 377-364, 1974.

a Padró Mossaguer, J., and cols. Liss lípidos en la alimentación parenteral. Medicina Clinica, 61: 260-266, 1973.

9 Dudrick, S. J. Wilmore, D. W., and Wars, H. M. Long term total parenteral nutrition with growth in puppies and pusitive nitrogen balance in putients. Surg. Forum. 18: 356-357, 1967.

10 Dudrick, S. J. Long term total parenteral nutrition with gruwth, develupment and positive nitrogen balance. Surgery 64: 134142. 1968.

11 Dudrick, S. J.; Pyd Wilmore, D. W. Lung term parenteral feeding. Hospital Praclice 3: 65.78, 1968.

12 Dudrick, S. J.; Groff Duller, B.; Wilmore, D. W. Long term venous catheterizetion in infants. Surgery. Gynecolugy and Obstetrics. 805-806, 1969.

13 Dudrick, S. J., and cols. Can intravenous feeding as the sob means of nutrition support gruwth in the child and restore weight loss in as adult? Transaction of southerm Surgical Association. Vul. LXXX, 1969 .

14 Dudrick, S. J, and cols. Intravenous Hyperalimentation. Med. Clin. N. Amer. 54: 577-589, 1970.

15 Dudrick, S. J.; Vors, H. M., and Rhoods, J. E. Growth of puppies receiving all nutritional requirements by vein; in Fortsclritte der Parenteralen Ernāhrung. pp. 16-18 (Pallas, Lochharn bei München, 1967 ).

16 Hull, R. L. Lise of trace elements in intravenous hyperalimen. tation sulutions. Am. J. Hosp. Pharm. 21: 759.761, 1974.

17 Ricour, $C$, and cols. Trace elements in infants on total parenteral autrition. Abstr. 10th Int. Congr. on Parenteral Nutrition, Kyoto 1975a, p. 236.

18 Howars, A. F., and Krasna, H. Tolal intravenous nutrition by peripheral vein in neonatad surgical patienıs. Pediatrica 52: 1420,1973 .

19 Winters, $A$. W. The body fluids in pediatrics. Little, Brown and Co. Boston, 1973.

20 Ruzu, F, y cols. Principiog y realización de la alimentación parenteral ed el niñe. Revista Ibys, N.・2, 1975.

2I Borresen, H. C.; Coran, A. C., and Knutrud, O. Metabulic resulis of parenteral feeding in neonatal surgery. Ann. Surg. 172: 291-301, 1970a.

122 Heird, $w^{\prime}, C$, and cols. Intravenous Alimentation in Pediatric Patients. J. Pediat. 80: 351-372, 1972b.

23 Johnson, D. C. 'l'otal intravenous nutrition in newborn surgical patients. A three-year perspective. J. Pediat. Surg. 5: 601-605, 1970.

24 Caran, $A$. G. The long-term intravenous feeding of infunts using periphera] veins. J. Pediat. Surg. B: $601-807,1973$.

25 Puri, P.; Guiney, E. J, and O'Donnell, B. Total parenteral feeding in infants using peripheral veins. Auchs. Dis. Childh. 50. 133-136, 1975 .

26 Wilmore, D. W., and Dudrick, S. J. Safe long-term yenous calheterizstion. Archs. Surg. Chicago 98: 256-258, 1969b.

27 Shenkin, A., and Wretlind, A. Parenteral Nutritiun. Wld. Rev. Nulr. Diel., Vol. 28, pp. 1-111 (Karger, Basel, 1978). 
28 Lee, $\boldsymbol{H}$. A. Parenteral nutrition in acute metabolic illness Acadenic Press, New York, 1974).

24 Chan, J. M. C., and cols. Hyperalimentation with Aminu-acid and casein hydrolysate solvions. Mechanism of acjesss. JAMA $200: 1700-1705.1972$.

30 Heird, W.C, and cols. Metabulic acidnsis resulting from intravenous alimentation mixtures conlaining syathetic amino-acids. New Engl. J, Med. 287: 943-948, 1972a.

3. Ziegler, E. E., and Fomun, S.J. Fluid intake, renal solute load and water balance in infancy. The J. of Pediatrics 78: 561-569, 1971.

32 Froeseh, E. R. The Metabolism of Glucose, its Endocrine CionIrul and Comparative uspext with tructose. sorbitad and sylitid metabulism. Chapter 2, p. 13 in "parezteral Nutrition in acute metabolic illness". H. A. Lee. Academic Press (Londun, 1974).

33 Schultis, K, and Geser, C. A. Clinical experiments on the use of carbohydrates in stress; in Meng and Law Parenteral Nutriion, pp. 134-148 Thomas, sprint field, 1970!.

34 Schiener, W. Adverge effects of xylitol in parenteral nutrition. Metabolism 20: 345-347, 1971.

35 Lipson, A.; Glassont, M., and Ablett, W. Tnal parenleral nutrition in Puediatric Patients. The Medical Juurnd of Austrulia 2: 524-528. 1974.

3o Halberg, D, Schwberth, $O$, and Wrettind, A. Experimental and clinical studies with fat emulsion for intravenuus nutrition. Nutr. Dist. B: 245-281, 1966.

37 Hakansson, I. Experience in kng-term studies on the nine intravenous fat emulsions in dogs. Nucr. Dist. 10: 54-76, 13\%8.

38 Filler, R. M.: Eraklis, A. Care uf the tritically ill child. Intravenous almentation. Pediatrics 46: $456-461$, 1970 .

39 Schenk, $K$ E. Aliznent aciún con sonda $y$ alimentaciún parenteral en medicina interna. Med. Klin. 144: 66-79, 1974.

40 Bour, H. Notions général es conctrnan l'alimentation de f'humme el indications d ane alimentarion artiticielle. La Revue de Practicien 24: 297-307, 1974

41 FAO/WHO Expent Grup: Protein requirements. FAO Nutr. Meet. Fep. Ser. N. '37, 1465.

42 Bergstrōm, $J_{\text {, and }}$ cols. Improvement of nitrogen balance in a uremic patient by the addition of histidine to essential aminoacid solutions given intraveauusly. Life Sci. 9: 787-794. 1970.

43 Wilkinson, W. R.; Flores, L. L., and Pagones, J. N. Growth ol miccourganisms in parenteral nutritional lavids. If Addative Hevien: Reprinted from Drug Intelligence and Ctinical Pharmacy. 7 : 226-231, 1973 .

44 Bumi, C.; Henkempyer, H., and Póssier, H, H. Katbeterembolism. Scheiz Med. Waschr. J0]: 1576-1577, 1971.

45 Keating, J. P.; Teruberg.J. L., and Gleasun, W. A. Parenteral Hyperalimentaion at Sit. Louis Childre's Huspital. Clivical Digest 2, N." 1, eneru, 1973.

16 Kaplan, M, arai cols. High caloric glucosa-nitrogen infusions. Arch. Surg. 99: 567.571 , 1969.

47 Kanarek, K. S.i Wolfsulorf. M. B., and Dinner, M. Hyperalimentation. A case report of its use in perforated necrozing anteruculitis. Medical Journal 5: 599-601, 1971.

43 Solassol, $C_{+}$, and cals. Nourelles techniques de nutrition parentérale a long terme gour suppléance intestinale. J. Chir., Paris 105: 15-24, 1973.

$4 y$ Parsa, H. M.; Beverty, H. T, and Ferrer, J. M. Central venous alimentation. American Joumal of Nursing 27: 2042-2047, 1972.

50 Parsa, $H . M$, and cols. Central venous nutrition with a crystaline L-aminuecid solution and hypertunic dextrose. Miraggafía Columbialniversity. New York.

51 Michener, $W$. M.; Raw, 0 . Parenteral Nutrition: the age of the catheter. Pediatric Clinies of North America. 17: 373-383, 1970.

52 Meng, H. K.; Soudstead, H. H. Total Parenteral Alimentation. Vanderbit Lnipersity and School of Medicinc. Nasville. Tennesse, 1970.

53 Rea, W. J., and cols. Intravenous Hyperosmolar Alimenlation. Arch. Surg. 100: 393-399, 1970.

54 Meng, H. C.; Lau, D. H., and Sundstread, H. H. Sume Clinical experience in Parenteral Nutrition. In advances in Parenteral Nutrition (G. Berg. ed.) p. 64, Georg Thieme Verlag, Stuttgart. 1969.

55 Parso, H.W. Nutrition in the superior cava with a salution of crystalline L-amino-acids and hypertunic dextrose. Monografí Columbia University Harlen Huspital Center, Surgieal Department. New Yurk.

56 O'Neill, 1. A. Werkshop N." b, delivery; in AMA Symp. Total Parenteral Yurrition. Nusville, 1972, pp. 252-257.

5 i Berdata, G. I. M., and Babson, S. G. Peripheral intravenirus alimentalion of the small premature intant. J. Pediat. 79: 494 498. 1971.

58 Grotte, $G$. Intravenous feeding in childhood. Postgrad. Med. 11: 104-132, 1973 .

5y Coran, A. G. Tolal intravenous feeding of infants and children without the use of a central venuus sutheter. Ano. Surg. 179: 45-449, 1974 .

dicutr, $C$; Millot, M, and Balsan, S. Phosphorus depletion in children on long-term total parenteral nulrition. Acta Pediat. Scand. 64: 345-392, 1975b.

ol Advances in Parenteral Nutrition. Ed. by Ivan D. A. Juhuston. MP Press Limiled-Lancaster. England, 1978.

b2 Saw,J.C. L. Nutrición parenteral en nî̀us enfermos con deficit de prso al macioniento. Cilinicins l’ediatríras de Ninteamérica (ed. espeñola), Mayo: 332-357, 1973.

63 Bentley, D. W, and Lepper, M. H. Septicemis related to indwelling venous catheter. J. Am. Med. Ass. 206: 1749-1752. 1968.

64. Kesster, E. Hyperalimentation in the management of gastroiuterlinal cutanesus fistulas. 5. Alr. J. sure. 12: 101-105, 1974.

65 Pilder, $\boldsymbol{h}$., and cols. Intravenous supplementation of L-aminoacids and dextrose in low-birth-weight infants. The Journal of Paliatrics. 42: 945-950, 1973.

t6 Pursa. H. M.; Ferrer.J. M., and Harbif, D. H. Sate and sterile maintenance of long-term centrai venous catheters. Monografia. Uctubre 1972.

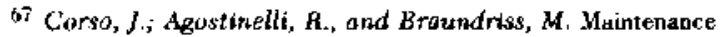
of Venuus Polyetbylene Catheters tu reduce riak of infection. JAMA 210: 2075-2077, 1969.

68 Parsa, H. M. Experiences with central venuss nutrition; problems and their prevention. Manografia de Columbia University, Collage of Physicians and surgeong. Surgical Service Harlem Hospital Center. New York. Ockubre, 1971.

69 Caloin, M. P., and cols. A safe lung-term infusion technique. The Lancel; 12: 317-320. 1972.

70 Dudrick, S. J. Parenteral Hyperalimentation. Metabolic Problems and Solutions. Annals of Surgery 176: N.*2, 1972.

7) Heird, W. C., and cols. Hyperammoneria tesuling from intravenuus alimeatation using a mixture of synthetic L-anino-acids. J. Pediat, 81: 162-165, 1972a.

72 Johnson, J. D.; Albitten, W. L., and Sunshire, F. Hyperammonetria accompanying parenteral nutrition in newbors inlant5. J. Pediat. 81: 154-161, 1972.

\section{RESUMEN}

Tras una revisión histónica se analizan las bases metabólicas de necesidades energéticus valorando los requerimientos de los tres principios inmediatos (hidratos de carbono, lipidos y proteinas), de oligoelementos y de vitaminas.

Se analizan ciertas peculiaridades metabólicas en la nutrición parenteral: Sobrecarga de solutos al rinón y ostnolaridad de las soluciones. Se detallan aspectos técnicos en la preparación y aportes de la nutrición parenteral (vía central y periférica). Se especifican los controles y manejo de la nutrición parenteral. Finalmente se detallan las complicaciones técnicas y metabólicas achacables a la nutrición parenteral. 